\title{
Ceftolozane-tazobactam in an elastomeric infusion device for ambulatory care: an in vitro stability study
}

\author{
Edward Raby (10 , 1,2 Saiyuri Naicker, ${ }_{1}^{3,4}$ Fekade Bruck Sime, ${ }^{3,4}$ Laurens Manning, 1,2 \\ Steven C Wallis, ${ }^{3}$ Saurabh Pandey, ${ }^{3}$ Jason A Roberts ${ }^{4,5}$
}

${ }^{1}$ Infectious Diseases

Department, Fiona Stanley Hospital, Murdoch, Western Australia, Australia ${ }^{2}$ Internal Medicine, University of Western Australia Faculty of Medicine Dentistry and Health Sciences, Crawley, Western Australia, Australia

${ }^{3}$ University Of Queensland Centre for Clinical Research, University of Queensland, Herston, Queensland, Australia ${ }^{4}$ Centre for Translational Antiinfective Pharmacodynamics, Pharmacy Australia Centre of Excellence, Brisbane, Queensland, Australia ${ }^{5}$ Pharmacy Department, Royal Brisbane and Women's Hospital, Herston, Queensland, Australia

\section{Correspondence to}

Dr Edward Raby, Infectious Diseases, Fiona Stanley Hospital, Murdoch WA 6150, Australia; edward.raby@ health.wa.gov.au

ER and SN contributed equally.

Preliminary results from this study were presented as a poster at the 20th Annual Scientific Meeting of the Australian Society for Antimicrobials, February 2019, Sydney, Australia.

Received 16 September 2019 Revised 22 November 2019 Accepted 4 December 2019 Published Online First 16 December 2019

\section{EAHP Statement 3:}

Production and Compounding.

\section{Check for updates}

(C) European Association of Hospital Pharmacists 2020. No commercial re-use. See rights and permissions. Published by BMJ.

To cite: Raby $E$, Naicker $S$, Sime FB, et al.

Eur I Hosp Pharm

2020;27:e84-e86.

\section{ABSTRACT \\ Objectives Published in vitro stability data for} ceftolozane-tazobactam supports intermittent short duration infusions. This method of delivery is not feasible for many outpatient antimicrobial therapy services that provide only one or two visits per day. This study aimed to assess time, temperature and concentrationdependent stability of ceftolozane-tazobactam in an elastomeric infusion device for continuous infusion across clinically relevant ranges encountered in outpatient antimicrobial therapy.

Methods Ceftolozane-tazobactam was prepared to achieve initial concentrations representing total daily doses for 'renal', 'standard' and 'high' dose schedules in elastomeric infusion devices with a volume of $240 \mathrm{~mL}$. Infusion devices incubated at room and body temperature were serially sampled over 48 hours. Refrigerated infusion devices were sampled over 10 days. Concentrations of ceftolozane and tazobactam were separately quantified using a validated ultra-high performance liquid chromatography-photodiode array method.

Results The greatest loss of ceftolozane occurred at $37^{\circ} \mathrm{C}$, however, stability remained above $90 \%$ at 24 hours. Tazobactam was more stable than ceftolozane under these conditions. There was minimal loss at $4^{\circ} \mathrm{C}$ for either component over 7 days.

Conclusions Ceftolozane-tazobactam is suitable for ambulatory care delivered as a continuous infusion via an elastomeric infusion device.

\section{INTRODUCTION}

Published in vitro stability data for ceftolozanetazobactam supports intermittent short duration infusions at a standard dose of $1.5 \mathrm{~g}$ three times a day. ${ }^{1}$ Thrice daily visits are not practical for many outpatient parenteral antimicrobial therapy (OPAT) services and measures to facilitate once daily visits, such as continuous 24-hours infusions are preferred. ${ }^{2}$ A recent case report demonstrated the clinical utility and likely stability of ceftolozanetazobactam administered as a continuous infusion by elastomeric infusion device. ${ }^{3}$ Elastomeric infusion devices are highly practical but present challenges to drug stability. They have a limited volume that may result in high drug concentrations and are often exposed to increased ambient temperature due to positioning close to the patient. ${ }^{45}$ In the current study, experimental conditions were established in vitro to assess time, temperature and concentration-dependent stability of ceftolozanetazobactam encompassing clinically relevant ranges for use as continuous infusion in OPAT, including a maximum dose of $9 \mathrm{~g}$ per day as proposed for nosocomial pneumonia. ${ }^{6}$

\section{METHODS}

Sample preparation and experimental conditions Ceftolozane-tazobactam powder for infusion (Merck Sharp \& Dohme, West Point, Pennsylvania, USA) was reconstituted as per the manufacturer's product information and then diluted in $0.9 \%$ sodium chloride for injection to achieve initial concentrations representing total daily doses for 'renal', 'standard' and 'high' dose schedules (table 1) in LV10 elastomeric infusion devices with a volume of $240 \mathrm{~mL}$ and intended infusion rate of $10 \mathrm{~mL} / \mathrm{h}$ (Baxter, Old Toongabbie, New South Wales, Australia).

Prepared infusates were incubated at body temperature $\left(37^{\circ} \mathrm{C}\right)$, room temperature $\left(25^{\circ} \mathrm{C}\right)$ or refrigerated at $4^{\circ} \mathrm{C}$. Body and room temperature infusates were protected from light during incubation; refrigerated infusates were out of direct sunlight. No precautions against ambient light exposure were taken during preparation or manipulation of samples. Five elastomeric infusion device replicates were prepared for each experimental condition (dose-temperature). Samples were taken at $0,6,18,22,24,30$ and 48 hours for all temperature conditions with additional samples taken at 72 , 120,168 and 240 hours for the $4{ }^{\circ} \mathrm{C}$ storage condition. Infusors were inverted three times prior to sampling and the first $100 \mu \mathrm{L}$ of infusate from the tubing was discarded.

\section{Physical assessments}

Samples were transferred to a glass vial for colour, clarity and precipitation assessments. Presence or absence of colour change was assessed visually for each sample held against a white card, in addition, colour photographs taken at each time point were compared with baseline (time 0 ). Clarity was reported as clear or turbid by comparison with a blank (drug free) $0.9 \%$ sodium chloride solution for injection against a striped card. Precipitants were reported as present or absent after visual inspection while agitating the vial. Subvisible particle detection was performed by dynamic light scatter on a $1 \mathrm{~mL}$ aliquot using the Zetasizer Nano (Malvern Instruments, Malvern, Worcestershire, UK) with a refractive index of 1.39 and an absorption coefficient of 0.1 over eight runs of $10 \mathrm{~s}$ each per sample at $25^{\circ} \mathrm{C}$. Presence of subvisible particles was reported if any particles were detected with a diameter in the order of $\geq 10000 \mathrm{~nm}$. A $0.25 \mathrm{~mL}$ aliquot was used to assess the $\mathrm{pH}$ using a LAQUA Twin compact $\mathrm{pH}$ 
Table 1 Dosing schedules and initial concentrations

\begin{tabular}{llllllc}
\hline \multirow{2}{*}{ Dosing schedule } & \multicolumn{3}{l}{ Total daily dose, $\mathbf{g}$} & & \multicolumn{2}{c}{ Concentration, g/L } \\
\cline { 2 - 4 } \cline { 7 - 7 } & C/T & CTZ & TZB & & CTZ & TZB \\
\hline Renal & 0.45 & 0.3 & 0.15 & & 1.25 & 0.63 \\
Standard & 4.5 & 3 & 1.5 & & 12.5 & 6.25 \\
High & 9 & 6 & 3 & 25 & 12.5 \\
\hline
\end{tabular}

C/T, ceftolozane-tazobactam combined; CTZ, ceftolozane; TZB, tazobactam.

meter (Horiba, Kyoto, Kyoto Prefecture, Japan) according to manufacturer's instructions.

\section{Drug concentrations}

Concentrations of ceftolozane and tazobactam were separately quantified using a validated method on a Nexera ultrahigh performance liquid chromatography system coupled to a photodiode array (PDA) detector (Shimadzu, Kyoto, Kyoto Prefecture, Japan). Separations were performed on an Xterra RP C18, $2.1 \times 150 \mathrm{~mm}(3.5 \mu \mathrm{m})$ analytical column (Waters, Milford, Massachusetts, USA) preceded by a SecurityGuard Ultra C18 guard cartridge (Phenomenex, Torrance, California, USA). Mobile phase composition was $87 \%$ phosphate buffer (0.1 M, pH 7) prepared using disodium hydrogen phosphate (Merck, Darmstadt, Hesse, Germany) and orthophosphoric acid (VWR International BDH, Radnor, Pennsylvania, USA) with $13 \%$ methanol. The mobile phase was delivered isocratically at $0.25 \mathrm{~mL} / \mathrm{min}$ and produced a backpressure of $3400 \mathrm{psi}$. The PDA monitored the UV spectrum from 190 to $400 \mathrm{~nm}$. Approximate retention times and wavelength for quantitation for each analyte peak were: ceftolozane $2.3 \mathrm{~min}$ at $310 \mathrm{~nm}$ and tazobactam $2.9 \mathrm{~min}$ at $225 \mathrm{~nm}$. Samples were quantified against a calibration curve generated from the batch calibrators.

\section{Statistical methods}

All data manipulation and analysis was conducted using RStudio (RStudio Team (2015). RStudio: Integrated Development for R. RStudio, Boston, Massachusetts, USA, http://www.rstudio. $\mathrm{com} /$ ). For stability analysis, concentrations were converted to percentage of baseline mean for each condition (dosetemperature). A simple linear regression model was fitted to assess the association between time and concentration. Potential outliers were identified by analysis of Cook's distance and removed if confirmed to be extreme on visual inspection. The non-linear least squares method was used to fit an exponential model to assess the effect of temperature on concentration with estimates of the mean predicted using the second order Taylor expansion from the predictNLS function (propagate v1.0-4, Spiess A-N). All percentage estimates are presented as mean with $95 \%$ CI. Stability was predefined as greater than $90 \%$ of the baseline mean remaining at 24 hours in the absence of visible or subvisible particles. The 30 and 48 -hours time points were included in the room and body temperature arms for analytical purposes to increase confidence in the 24-hours estimate and should not be used as clinical validation or endorsement of continuous infusion beyond 24 hours.

\section{RESULTS}

All samples were free of sub-visible and visible particulate matter. There were no observed changes in clarity or colour in any of the refrigerated $\left(4^{\circ} \mathrm{C}\right)$ samples or at 24 hours in the samples from elastomeric infusion devices incubated at room $\left(25^{\circ} \mathrm{C}\right)$ or body $\left(37^{\circ} \mathrm{C}\right)$ temperature. An increase in intensity of yellow colour

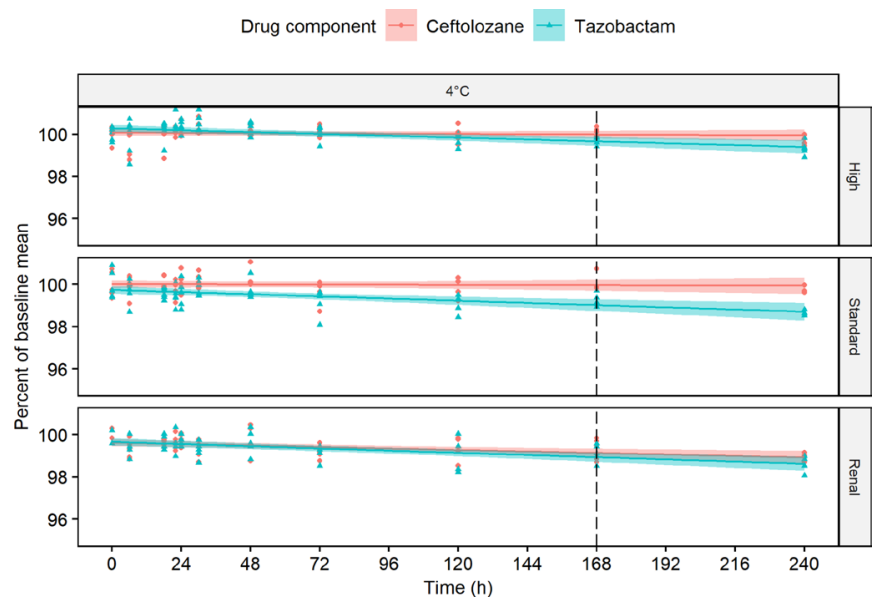

Figure 1 Scatter plot of ceftolozane (circles) and tazobactam (triangles) concentrations expressed as per cent of baseline mean against time for each of five replicates at renal, standard and high dosing schedules refrigerated at $4^{\circ} \mathrm{C}$. Fitted regression lines are plotted with shaded area showing $95 \% \mathrm{Cl}$. Dotted vertical line identifies the critical time point at 7 days.

was observed at 48 hours for the room and body temperature samples. The $\mathrm{pH}$ was observed to fall over time under every condition tested with the maximal drop being from 5.8 to 5.5 at 24 hours in the high dose elastomeric at body temperature. All samples remained within the $\mathrm{pH}$ range 5.5-6.2.

Tazobactam showed similar stability to ceftolozane when refrigerated (figure 1) and at room temperature (figure 2) and was clearly more stable at body temperature (figure 2). Ceftolozane met the predefined stability criteria with $97.7 \%$ (97.6 to 97.9) remaining at 24 hours at room temperature for the renal dose, $97.6 \%$ (97.5 to 97.8) for the standard dose and $98.8 \%$ (98.7 to 98.9 ) for the high dose. Ceftolozane stability was also maintained at 24 hours when incubated at body temperature with $93.0 \%$ (92.4 to 93.6) for the renal, $93.3 \%$ (93.1 to 93.4) for the standard and 93.2\% (93.0 to 93.4) for the high dose.

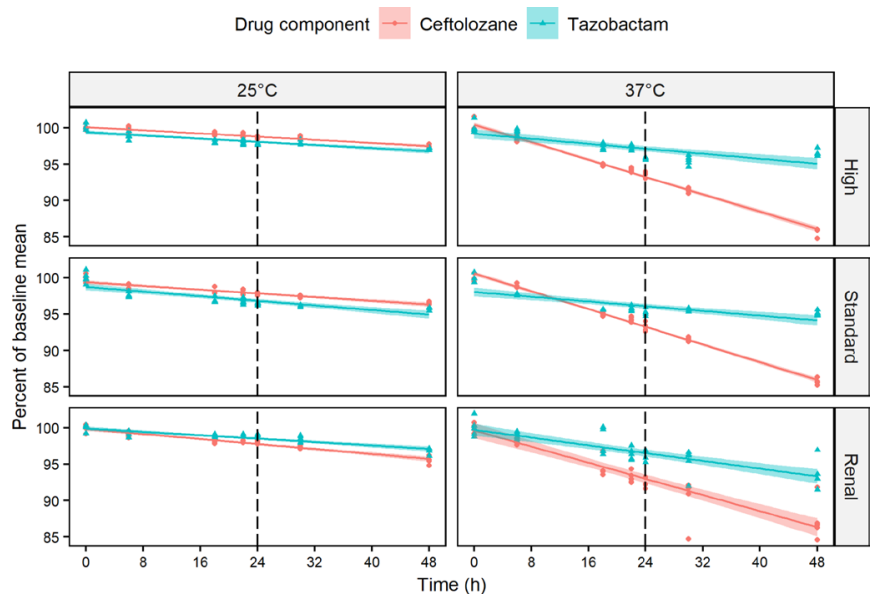

Figure 2 Panel of scatter plots showing ceftolozane (circles) and tazobactam (triangles) concentrations expressed as per cent of baseline mean against time for each of five replicates at renal, standard and high dosing schedules incubated at either $25^{\circ} \mathrm{C}$ representing room temperature or $37^{\circ} \mathrm{C}$ for body temperature. Fitted regression lines are plotted with shaded area showing $95 \% \mathrm{Cl}$. Dotted vertical line identifies the critical time point. 


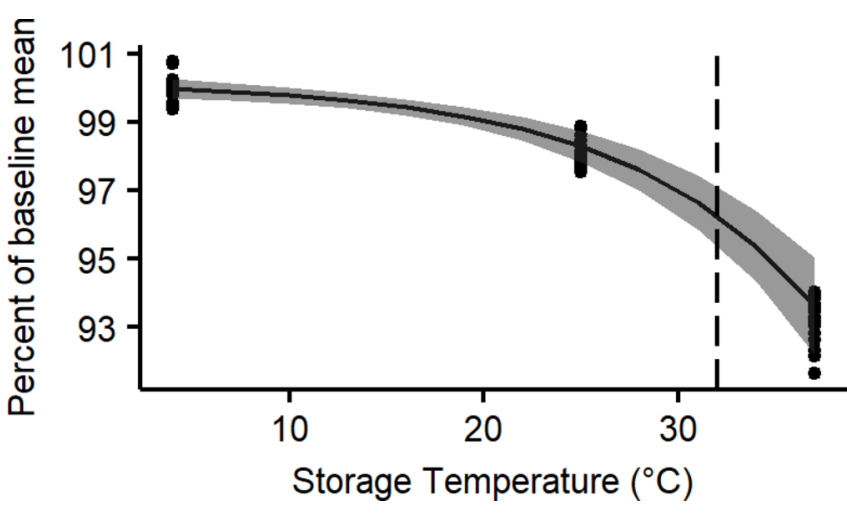

Figure 3 Scatter plot of ceftolozane concentrations expressed as per cent of baseline mean against storage temperature at 24 hours. Fitted exponential regression line is plotted with shaded area showing $95 \% \mathrm{Cl}$. Dotted vertical line identifies the critical temperature of $32^{\circ} \mathrm{C}$.

The relationship between temperature and ceftolozane per cent remaining at 24 hours is shown in figure 3 , as data points were seen to cluster, pooled data from the three doses were used to fit an exponential model which gave an estimate for $32^{\circ} \mathrm{C}$ as $96.2 \%$ (95.3 to 97.2). There was minimal loss at $4^{\circ} \mathrm{C}$ with $99.1 \%$ (98.9 to 99.3 ) ceftolozane remaining at 7 days (168 hours) for renal, $99.9 \%$ (99.7 to 100.2 ) for standard and $100.0 \%(99.8$ to 100.2) for high dose preparations (figure 1).

\section{DISCUSSION}

Using methods that are consistent with recent recommendations, ${ }^{2}$ elastomeric infusion devices for ambulatory care containing a total daily dose of ceftolozane-tazobactam in $240 \mathrm{~mL}$ remain stable for 24 hours at $21^{\circ} \mathrm{C}$ and $37^{\circ} \mathrm{C}$. There were no appreciable concentration-related effects on drug stability. It should be noted that we used the $90 \%$ stability standard as defined in the European and US Pharmacopoeias, however the most recent UK yellow cover document introduced a 95\%-105\% imit. $^{7}$ Although falling below $95 \%$ at $37^{\circ} \mathrm{C}$, our modelling suggests acceptable stability at $32^{\circ} \mathrm{C}$. The temperature of devices has been shown to vary depending on proximity to the body and placement relative to clothes and bedclothes, ${ }^{45}$ however no sustained periods above $32^{\circ} \mathrm{C}$ have been documented and the temperature of the infusate is likely to be well below this for much of the day. We have also demonstrated stability of ceftolozane-tazobactam in these devices when refrigerated for up to 7 days.

The main limitation of this study is that we did not attempt to identify the nature of any breakdown products. We did observe a fall in $\mathrm{pH}$ in parallel with loss of ceftolozane which is intriguing as ceftolozane alone has a $\mathrm{pH}$ of $2.0^{8}$; it remains unclear if this is due to an accumulating acidic breakdown product or degradation of the solution buffer. The changes in $\mathrm{pH}$ were not considered to represent a clinically significant stability issue. ${ }^{7}$ Another limitation is that we have only investigated a single elastomeric infusion device and due to potential interaction with the drug contact surface these results cannot necessarily be extrapolated to other infusion devices. We also did not conduct sequential temperature studies but the cumulative loss from 7 days of refrigeration and then 24 hours of ambulatory temperature is likely to be acceptable. It should be noted that the product information recommends vials be protected from light, but as this warning is not applied to the reconstituted solution, we did not explicitly control light exposure. Any effect of light has not been described in published literature and this could be investigated if aiming for a higher stability target.

In conclusion, ceftolozane and tazobactam are both stable across a range of clinically relevant temperatures and concentrations in a $240 \mathrm{~mL}$ elastomeric infusion device. Ceftolozane-tazobactam is considered suitable for ambulatory care to deliver total daily doses as a continuous infusion.

\section{What this paper adds}

What is already known on this subject

- Ceftolozane-tazobactam is stable for delivery when dosed by intermittent infusion.

- Elastomeric infusion devices provide convenient delivery of antibiotics as a continuous infusion in the outpatient setting.

- Delivering total daily dose as a continuous infusion presents challenges for stability due to the need of high drug concentrations and potential prolonged exposure to body temperature.

\section{What this study adds}

- Ceftolozane and tazobactam are both stable across a range of clinically relevant temperatures and concentrations in a $240 \mathrm{~mL}$ elastomeric infusion device.

Twitter Saiyuri Naicker @Sai_Naicker

Contributors All listed authors fulfil the 4 ICMJE criteria, there have been no other contributors.

Funding Supported in part by a research grant from the Investigator Initiated Studies Program of Merck Sharp \& Dohme (Australia) Pty Ltd [IIS 57279].

Disclaimer The opinions expressed in this paper are those of the authors and do not necessarily represent those of Merck Sharp \& Dohme (Australia) Pty Ltd.

Competing interests JR and LM have participated as MSD expert advisory board members and JR has undertaken consultancies for MSD. The other authors have no conflicts of interest to declare.

Patient consent for publication Not required.

Provenance and peer review Not commissioned; externally peer reviewed.

Data availability statement Original data in an excel spreadsheet are available on reasonable request from the corresponding author (ORCID iD 0000-0003-16716188).

\section{ORCID iD}

Edward Raby http://orcid.org/0000-0003-1671-6188

\section{REFERENCES}

1 Terracciano J, Rhee EG, Walsh J. Chemical stability of Ceftolozane/Tazobactam in Polyvinylchloride bags and elastomeric pumps. Curr Ther Res Clin Exp 2017;84:22-5

2 Jenkins A, Hills T, Santillo M, et al. Extended stability of antimicrobial agents in administration devices. J Antimicrob Chemother 2017;72:1217-20.

3 Stewart A, Roberts JA, Wallis SC, et al. Evidence of clinical response and stability of Ceftolozane/Tazobactam used to treat a carbapenem-resistant Pseudomonas aeruginosa lung abscess on an outpatient antimicrobial program. Int J Antimicrob Agents 2018;51:941-2.

4 Manning L, Wright C, Ingram PR, et al. Continuous infusions of meropenem in ambulatory care: clinical efficacy, safety and stability. PLoS One 2014;9:e102023.

5 Voumard R, Van Neyghem N, Cochet C, et al. Antibiotic stability related to temperature variations in elastomeric pumps used for outpatient parenteral antimicrobial therapy (OPAT). J Antimicrob Chemother 2017;72:1462-5.

6 Xiao AJ, Miller BW, Huntington JA, et al. Ceftolozane/tazobactam pharmacokinetic pharmacodynamic-derived dose Justification for phase 3 studies in patients with nosocomial pneumonia. J Clin Pharmacol 2016;56:56-66.

7 NHS Pharmaceutical Quality Assurance Committee. Standard protocol for deriving and assessment of stability, part 1: aseptic preparations (small molecules. 4th edn, 2017

8 Sharp M, Corp D. Zerbaxa (ceftolozane and tazobactam) [prescribing information]. Whitehouse Station, NJ, USA, 2015. 